\title{
Estresse em mães de crianças com asma: relato de pesquisa de um programa de redução do estresse
}

\author{
Stress in mothers of asthmatic children: research report of a stress reduction program \\ Estrés en madres de niños asmáticos: relato de investigación de un programa de \\ reducción del estrés
}

\author{
Sandra Cairo de Oliveira Amaral ${ }^{1}$, Clemax Couto Sant'Anna ${ }^{1}$
}

\begin{abstract}
RESUMO
Objetivo: Relatar o impacto de um programa para redução do estresse em mães de crianças com asma atendidas em um ambulatório público de pneumologia do Rio de Janeiro. Metodologia: Estudo prospectivo quase-experimental e longitudinal com abordagem quantitativa, incluindo quatro mães de crianças com asma. $O$ programa teve duração de quatro sessões semanais de 1 hora e 30 minutos cada. Foi utilizado o Inventário de Sintomas de Stress para Adultos de Lipp (ISSL) para avaliação das fases do estresse em três momentos: préteste, pós-teste e 8 meses após a finalização do programa. Resultados: Foi observado decréscimo nos níveis de estresse das participantes durante o estudo. Conclusão: A participação das mães no programa pode influenciar positivamente nos níveis de estresse e os resultados observados reforçaram a importância da implementação de programas de intervenção para controle e redução de estresse para essa população.
\end{abstract}

Palavras-chave: Estresse Psicológico, Mães, Crianças, Asma.

\begin{abstract}
Objective: To report on the impact of a stress reduction program among mothers of children with asthma who were cared for at a pneumology outpatient clinic in Rio de Janeiro. Methods: Prospective, longitudinal, quasiexperimental study with a quantitative approach that included four mothers of children with asthma. The program had four weekly sessions, each lasting 1 hour and 30 minutes. We used Inventário de Sintomas de Stress de Lipp (LSSI) to evaluate the stress phases at three moments: pre-test, post-test and 8 months after the end of the program. Results: We observed a decrease in the stress levels of participating mothers during the study. Conclusion: Mothers' participation in the program may positively influence their stress levels. Study results reinforced the importance of implementing intervention programs for controling and recuding stress among this population.
\end{abstract}

Keywords: Psychological Stress, Mothers, Children, Asthma.

\section{RESUMEN}

Objetivo: Informar el impacto de un programa para reducir el estrés en madres de niños con asma atendidas en un ambulatorio público de neumología de Río de Janeiro. Metodología: Estudio prospectivo casi experimental y longitudinal con abordaje cuantitativo, incluyendo cuatro madres de niños con asma. El programa tuvo una duración de cuatro sesiones semanales de 1 hora y 30 minutos cada una. Se utilizó el Inventario de Síntomas de Tensión para Adultos de Lipp (ISSL) para evaluar las fases del estrés en tres momentos: pre-test, post-test y 8 meses después de la finalización del programa. Resultados: Se observó un descenso en los niveles de estrés de las participantes durante el estudio. Conclusión: La participación de las madres en el programa puede influenciar positivamente en los niveles de estrés y los resultados observados reforzaron la importancia de la implementación de programas de intervención para control y reducción de estrés para esa población.

Palabras clave: Estrés Psicológico, Madres, Niños, Asma. ${ }^{1}$ Universidade Federal do Rio de Janeiro (UFRJ), Rio de Janeiro-RJ. E-mail: cairosandra@gmail.com
Financiado pela CAPES - Coordenação de Aperfeiçoamento de Pessoal de 


\section{INTRODUÇÃO}

Considerada um dos problemas mundiais de saúde, a asma é uma das condições crônicas mais comuns e ocorre em pessoas de todas as idades (DSBP, 2012; GLOBAL ASTHMA REPORT, 2014), sendo a doença crônica não transmissível mais comum da infância e adolescência (KUSCHNIR et al., 2016). A existência da asma infantil pode provocar possíveis consequências não apenas para a criança, mas também para o seu meio familiar (CASTRO e PICCININI, 2002). No caso da asma, a necessidade de cuidados diários e contínuos constitui uma preocupação constante para os pais, assim como, a sobrecarga aos cuidadores pode ser considerada um estressor (CAIRO e SANT'ANNA, 2014).

O estresse, definido como uma reação psicofisiológica complexa que se inicia da necessidade do organismo manter seu equilíbrio, passa a ser considerado e reconhecido como fator de risco em estudos sobre a asma (LIPP, 2003). Estudos apontam que o estresse pode influenciar o risco de episódios de chiado, contribuir para o aparecimento e agravamento das crises de asma, além disso, o alto nível de estresse familiar poder aumentar a produção inflamatória nas crianças e os sintomas de asma (WRIGHT et al., 2002; KAUGARS et al., 2004; BELLIN et al., 2013; YEH et al., 2016).

No cuidado da criança com asma, acredita-se que as mães são representantes do papel principal de cuidador e o membro da família que faz mais acomodações em termos de papéis e de tempo dedicados aos cuidados ao filho (CROWE et al., 2000; MATSUKURA et al., 2007). São elas que geralmente acompanham as crianças as consultas e vão com mais frequência aos hospitais, recebem instruções sobre a utilização de medicação e se envolvem mais no processo de tratamento (SILVER et al., 1996; PARENTE et al, 2012). A demanda de cuidados da criança acaba por tornar as mães cuidadoras alvos potenciais de diferentes estressores e mais vulneráveis ao estresse (SALOMÃO Jr, et al., 2008).

Estudos demonstram que os resultados da asma pediátrica são afetados pelo estresse psicológico do cuidador e acredita-se que programas de intervenção contribuam para a redução e controle do estresse desses cuidadores (CELANO, et al., 2012; YEH et al., 2016). Apesar da literatura internacional apresentar resultados positivos em relação a intervenções para redução do estresse em mães e cuidadores de crianças com doenças crônicas (incluindo a asma), a literatura brasileira não apresenta estudos de intervenção para a redução do estresse direcionado a cuidadores e mães de crianças com asma. Diante desse panorama, acredita-se que desenvolver intervenções que venham contribuir nesse campo é de fundamental importância para a saúde das cuidadoras.

O objetivo desse estudo, portanto, consistiu em relatar o impacto de um programa para redução dos níveis de estresse em mães de crianças com asma.

\section{MÉTODOS}

Trata-se de um estudo prospectivo quase-experimental e longitudinal com abordagem quantitativa.

Participaram do estudo quatro mães de crianças com diagnóstico de asma atendidas no Ambulatório de Pneumologia do Hospital Pediátrico da Universidade Federal do Rio de Janeiro. Todas as mães apresentavam níveis de estresse identificado através de instrumento de mensuração de estresse e participaram inicialmente do estudo sobre estresse e estressores nessa mesma instituição. Tal estudo intitulado "Estresse em mães e cuidadoras de crianças e adolescentes com asma: um estudo sobre a frequência do estresse e fatores estressores" teve como objetivo descrever a frequência do estresse e identificar fatores estressores nessa população (CAIRO e SANT'ANNA, 2014).

Foram utilizados os instrumentos de coleta de dados, Critério de Classificação Econômica Brasil e Inventário de Sintomas de Stress para Adultos de Lipp (ISSL). O ISSL avalia a presença dos sintomas de estresse em adultos, a fase do estresse e a porcentagem na fase. $O$ instrumento segue $O$ Modelo Quadrifásico desenvolvido pela psicóloga brasileira Marilda Lipp a qual propõe que o processo do estresse se desenvolve em quatro fases: fase inicial denominada fase de Alerta, seguida da fase de Resistência, 
fase de Quase-Exaustão e por último a fase de Exaustão (LIPP, 2005). O instrumento foi aplicado no primeiro e último dia do programa e 8 meses após da finalização do programa.

As mães assinaram o Termo de Consentimento Livre e Esclarecido e o estudo foi realizado após a aprovação do Comitê de Ética em Pesquisa da instituição (CAAE: 30427814.3.0000.5264) de 07 de maio de 2014.

A pesquisa teve início em janeiro de 2015 (Pesquisa 2015) com a definição da amostra e delineamento do conteúdo do programa, a realização do programa de redução do estresse com as quatro participantes ocorreu no período de junho e julho de 2015.

\section{Programa}

O programa foi realizado no Hospital Pediátrico, em sala reservada. Teve a duração de 4 sessões semanais de 1 hora e meia cada. O programa foi elaborado através de vasta pesquisa da literatura sobre 0 tema e as sessões foram baseadas em trabalhos de intervenções para redução e controle do estresse em mulheres, mães, familiares e cuidadores de crianças com doenças crônicas (incluindo asma) que apresentaram resultados significativos em relação à atenuação do estresse (TANGANELLI, 2003; WARNER et al., 2011; SABMANN et al., 2012; MAHMOUD e ELAZIZ, 2015; HENDRICKS-FERGUSON et al., 2017). O programa teve foco psicoeducativo, abordando temas como a asma infantil e técnicas voltadas para a redução e controle do estresse. Todas as sessões foram conduzidas por uma psicóloga e a sessão dedicada a informações sobre a asma infantil (sessão 3) teve a participação do Pneumologista Pediátrico responsável pelo ambulatório. A Quadro 1 descreve os temas e atividades desenvolvidas em cada sessão.

Todas as sessões envolveram técnicas de relaxamento e, ao final de cada prática, as mães relatavam sua experiência, avaliando os efeitos proporcionados pelo exercício realizado. As participantes eram incentivadas a praticarem os exercícios em casa, sendo combinado previamente com cada mãe os dias e horários que seriam realizados. Ao início de cada sessão (exceto a primeira), as mães eram convidadas a relatar sua prática durante a semana.

Quadro 1. Conteúdo por sessão do programa de redução de estresse para mães de crianças com asma.

\begin{tabular}{|c|c|}
\hline $\begin{array}{l}\text { Sessã } \\
0\end{array}$ & Tema / Atividade \\
\hline 1 & $\begin{array}{l}\text { Funcionamento do estresse. } \\
\text { Conceitos básicos do estresse e identificação dos estressores. Modelo Cognitivo do } \\
\text { Estresse. Sintomatologia do estresse. } \\
\text { Técnica de Relaxamento. }\end{array}$ \\
\hline 2 & $\begin{array}{l}\text { Os papéis desempenhados pela mulher. } \\
\text { Papéis desempenhados pela mulher no mundo atual (papel de mãe, esposa, profissional, } \\
\text { etc). O papel de mãe e cuidadora de uma criança com asma (cuidados em relação à } \\
\text { criança, sobrecarga do cuidado). } \\
\text { * Exercício de Respiração Profunda e Atenção Plena. }\end{array}$ \\
\hline 3 & $\begin{array}{l}\text { A asma na criança e no adolescente. } \\
\text { * Informações sobre a asma infantil (manifestações clínicas da doença, limitações } \\
\text { provocadas pela doença, medicação e tratamento). } \\
\text { Relaxamento Muscular Progressivo. }\end{array}$ \\
\hline 4 & $\begin{array}{l}\text { Plano para controle e redução do estresse. } \\
\text { Verificação das mudanças individuais percebidas em relação ao estresse (controle e } \\
\text { redução). Elaboração de um Plano Pessoal para Redução e Controle do Estresse com o } \\
\text { objetivo de continuidade da prática das técnicas e prevenção de recaídas. Relaxamento } \\
\text { para melhorar a autoestima. Avaliação do programa. }\end{array}$ \\
\hline
\end{tabular}

Fonte: Dados da Pesquisa, 2015.

REAS/EJC | Vol.11(1) | DOI: https://doi.org/10.25248/reas28_2019 Página 2348 de 2353 
Além da avaliação das técnicas realizada em cada sessão, na última sessão foi dedicado momento para avaliação geral do programa, onde as mães relataram sua experiência em relação a sua participação e realização dos exercícios. Após oito meses da finalização do programa, as mães foram contatadas para acompanhamento e questionadas sobre a prática das técnicas aprendidas durante sua participação no programa.

\section{RESULTADOS E DISCUSSÃO}

Com relação à participação das mães nas sessões, uma mãe teve 100\% de presença, sendo que 3 mães participaram 75\% das sessões. Tais ausências foram justificadas à psicóloga por telefone.

As mães apresentaram idade média de 37,75 anos $(D P=5,05)$ e a idade média dos filhos foi de 9,50 anos ( $D P=1,91)$, sendo $75 \%$ das crianças do sexo masculino. Em relação ao quadro de asma das crianças, $75 \%$ das crianças apresentavam asma leve no início do programa e $25 \%$ apresentavam asma moderada. Informações sobre as variáveis sociodemográficas encontram-se na tabela 1.

Tabela 1. Descrição das variáveis sociodemográficas de cada mãe.

\begin{tabular}{lccccc}
\hline \multicolumn{1}{c}{ Variáveis } & Mãe 1 & Mãe 2 & Mãe 3 & Mãe 4 \\
\hline Idade da mãe (anos) & 44 & 32 & 39 & 36 \\
\hline Estado civil & casada & casada & solteira & viúva \\
\hline Número de filhos & 1 & 1 & 3 & 1 \\
\hline Escolaridade & $\begin{array}{c}\text { fundamental } \\
\text { incompleto }\end{array}$ & médio completo & $\begin{array}{c}\text { fundamental } \\
\text { incompleto }\end{array}$ & médio completo \\
\hline Situação profissional & do lar & do lar & empregada & aposentada \\
\hline Renda familiar (em sm) & de 1 a 2 sm & de 1 a 2 sm & de 1 a 2 sm & de 1 a 2 sm \\
\hline $\begin{array}{l}\text { Idade da criança / } \\
\text { adolescente (anos) }\end{array}$ & 8 & 12 & 8 & 10 \\
\hline $\begin{array}{l}\text { Sexo da criança / } \\
\text { adolescente }\end{array}$ & masculino & masculino & masculino & feminino \\
\hline \begin{tabular}{l} 
Classificação da asma \\
\hline
\end{tabular} & persistente leve & persistente leve & $\begin{array}{c}\text { persistente } \\
\text { moderada }\end{array}$ & persistente leve \\
\hline
\end{tabular}

Fonte: Dados da Pesquisa, 2015. Abreviações: sm; salário mínimo

Em relação aos resultados sobre o nível de estresse apresentado pelo ISSL, antes da realização do programa, as quatro mães apresentavam presença de estresse. Observou-se que no início do programa a maioria das mães (75\%) encontrava-se na fase 2 (Resistência), diferindo apenas na porcentagem nessa fase, e uma das mães (25\%) encontrava-se na fase 3 (Quase-Exaustão), indicando maior nível de estresse. Após a realização do programa, uma mãe não apresentou estresse $(25 \%)$, duas mães permaneceram na fase 2 do estresse (50\%), porém, tiveram redução nas porcentagens nessa fase e uma mãe (25\%) permaneceu na mesma fase do estresse identificada no início do programa (Quase-Exaustão), entretanto, apresentou acréscimo na porcentagem dentro dessa fase. Após 8 meses da finalização do programa, duas mães não apresentaram níveis de estresse (50\%), uma mãe (25\%) manteve o resultado permanecendo na fase 2 (Resistência) e outra mãe (25\%) apresentou diminuição da fase do estresse da fase 3 (QuaseExaustão) para fase 1 (Alerta), conforme apresentado na tabela 2. 
Tabela 2. Descrição das fases e porcentagens do estresse nos três momentos (pré, pós, follow-up) para cada mãe.

\begin{tabular}{c|c|c|c|c}
\hline \multirow{2}{*}{ Momento } & \multicolumn{4}{|c}{ Fases do estresse e \%* } \\
\hline \multirow{2}{*}{ Pré-teste } & Mãe 1 & Mãe 2 & Mãe 3 & Mãe 4 \\
& $\begin{array}{c}\text { Resistência } \\
(25 \%)\end{array}$ & $\begin{array}{c}\text { Resistência } \\
(50 \%)\end{array}$ & $\begin{array}{c}\text { Resistência } \\
(42 \%)\end{array}$ & $\begin{array}{c}\text { Quase-Exaustão } \\
(67 \%)\end{array}$ \\
\hline \multirow{2}{*}{ Pós-teste } & Sem estresse & $\begin{array}{c}\text { Resistência } \\
(17 \%)\end{array}$ & $\begin{array}{c}\text { Resistência } \\
(8 \%)\end{array}$ & $\begin{array}{c}\text { Quase-Exaustão } \\
(83 \%)\end{array}$ \\
\hline \multirow{2}{*}{ Follow-up } & Sem estresse & Sem estresse & Resistência & Alerta \\
& & & $(8 \%)$ & $(78 \%)$ \\
\hline
\end{tabular}

${ }^{*}$ O valor da \% é uma medida conjugada com a fase do estresse, não uma medida isolada.

Fonte: Dados da Pesquisa, 2015.

O gráfico 1 ilustra a frequência das fases do estresse (em porcentagem) nos três momentos (pré, pósteste e follow-up).

O gráfico 2 ilustra as fases para cada mãe individualmente ao longo do programa.

Gráfico 1 - Frequência das fases do estresse em cada momento (pré, pós-teste e follow-up).

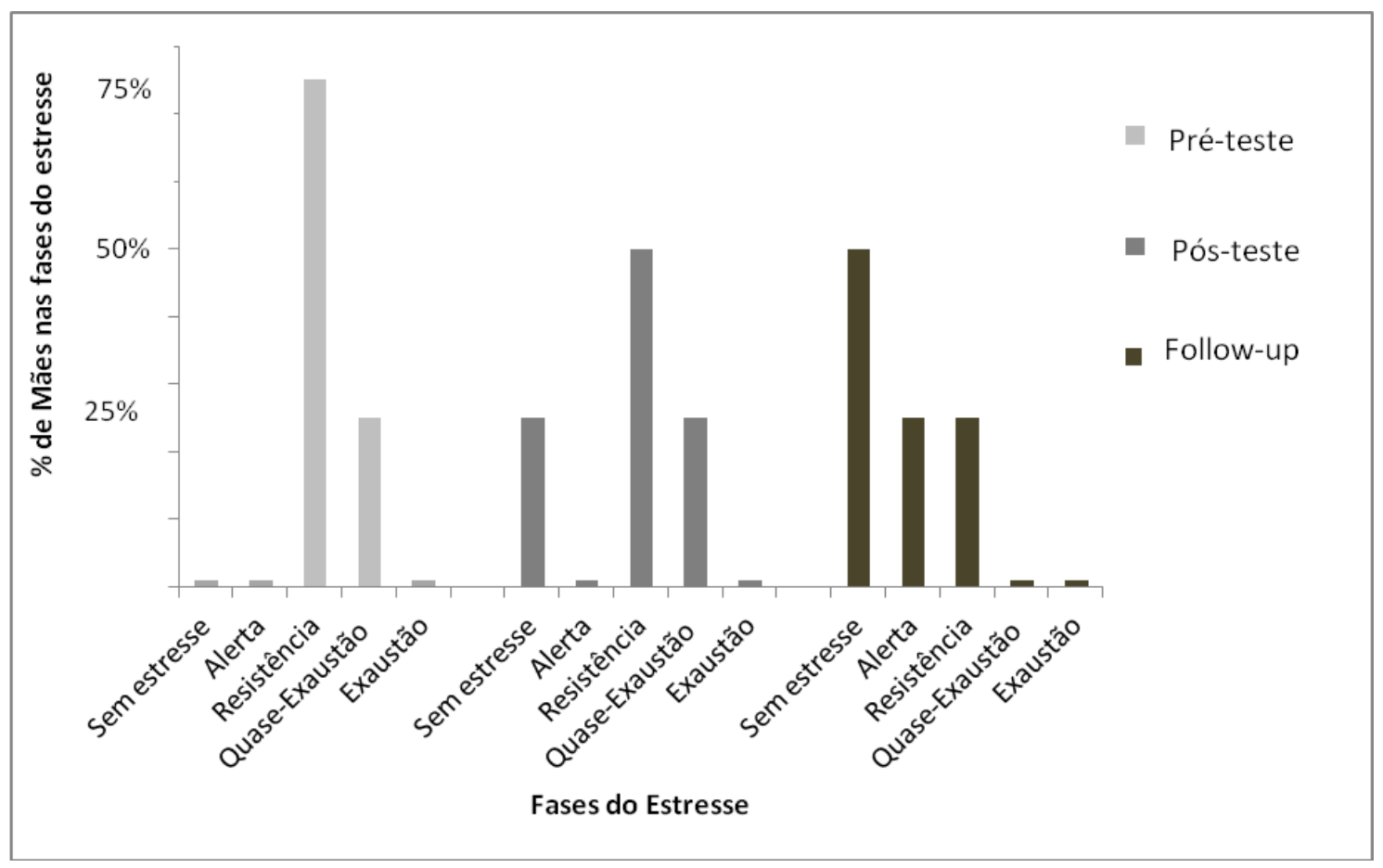

Fonte: Dados da Pesquisa, 2015. 
Gráfico 2 - Descrição das fases do estresse para cada participante nos três momentos.

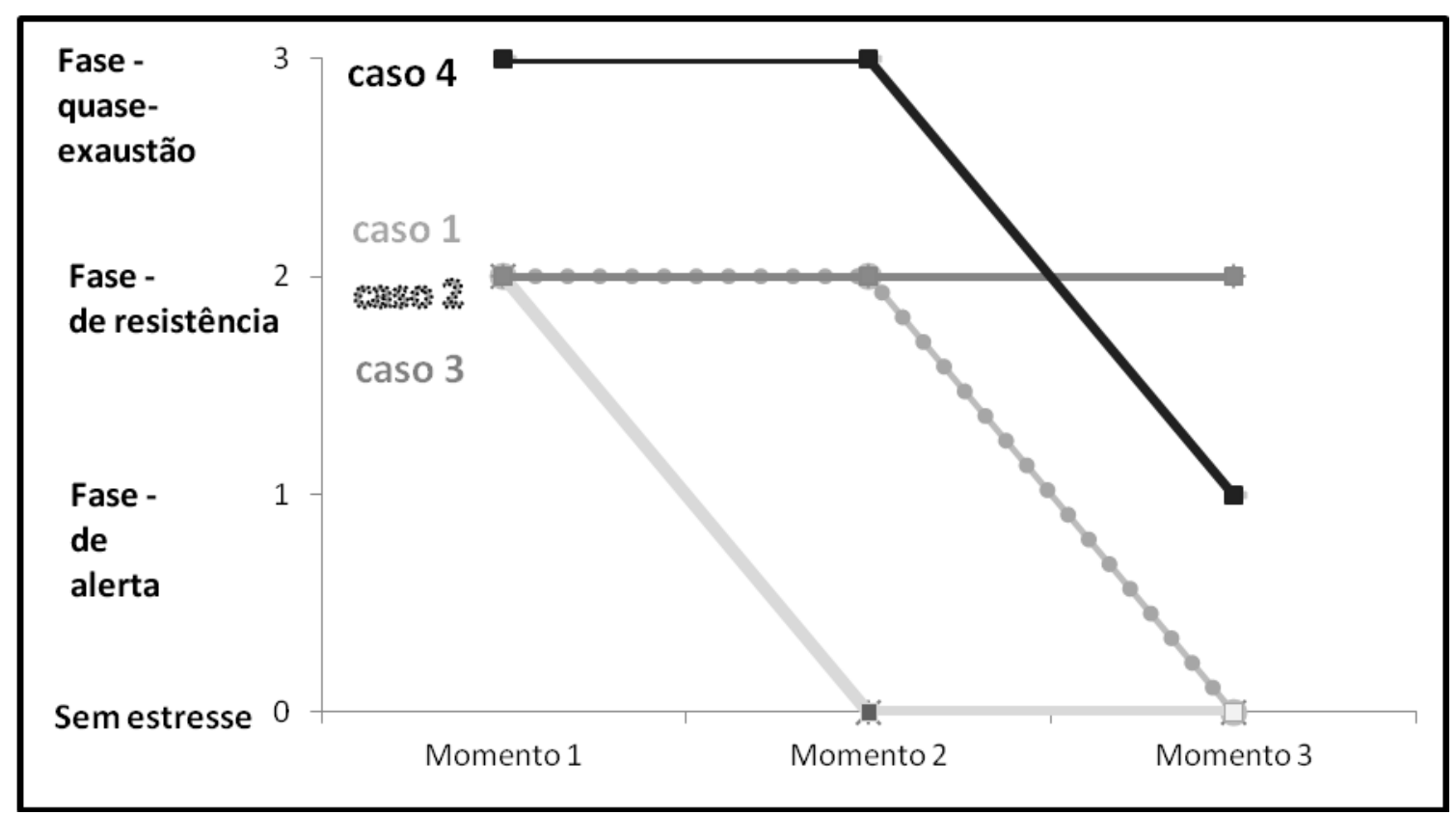

Fonte: Dados da Pesquisa, 2015. Legenda: Caso 1, Mãe 1; Caso 2, Mãe 2; Caso 3, Mãe 3; Caso 4, Mãe 4; Momento 1, pré-teste; Momento 2, pós-teste; Momento 3, follow-up.

Tais resultados apontam que as quatro mães apresentaram mudanças nos níveis de estresse do início do programa até o momento do follow-up. Observou-se que a Mãe 1 e a Mãe 2 iniciaram a intervenção na fase 2 (Resistência) e 8 meses após a finalização do programa (follow-up) não apresentaram níveis de estresse. Embora a Mãe 3 tenha permanecido durante os três momentos na mesma fase do estresse (Resistência), houve diminuição na porcentagem dessa fase do momento pré para o pós-teste, mantendo essa porcentagem até o momento do follow-up. A Mãe 4, portanto, iniciou o programa na fase 3 (Quaseexaustão), apresentou um aumento da porcentagem nessa fase no momento do pós-teste, porém, no período de 8 meses apresentou-se na fase 1 (Alerta), alterando da fase 3 para a fase 1 do estresse. Apesar do caráter exploratório, o estudo pode demonstrar que as mães apresentaram decréscimo nos níveis de estresse após a participação no programa. Esses resultados corroboram com estudos que relatam redução nos níveis de estresse de cuidadores de crianças com asma e outras doenças crônicas após participarem de programas de intervenção (MINOR, et al. 2006; WARNER et al., 2011; CELANO et al. 2012; SABMANN et al., 2012; TSIOULI et al., 2014; YEH et al., 2016). Dessa forma, os resultados desse programa demonstraram que a participação das mães pode influenciar positivamente na redução dos níveis de estresse, porém, tais níveis foram apresentados de forma muito singular. Isso reforça a necessidade de considerar nas pesquisas sobre estresse a influência de fatores biopsicossociais e a percepção sobre as fontes geradoras de estresse, assim como, fatores que envolvam a asma do filho (MATSUKURA et al, 2007). Segundo estudo de Cairo e Sant'Anna (2014) que identificou estressores de cuidadoras de crianças com asma, fatores emocionais, socioeconômicos e familiares, além da presença da doença no filho, foram identificados pelas mães como fatores geradores de estresse.

Após a realização do estudo, as mães que continuaram apresentando níveis de estresse (Mãe 3 e Mãe 4), foram orientadas e encaminhadas para acompanhamento psicológico.

A realização desse programa teve como objetivo oferecer estratégias de manejo do estresse, além de oferecer espaço para as mães compartilharem seus sentimentos e preocupações, conversarem sobre a doença do filho e sobre a sobrecarga do cuidado. A participação no grupo proporcionou a criação de uma rede social entre as quatro mães que continuou após o programa, com a criação um grupo virtual. Segundo 
Pellitier et al. (1994), mães de crianças cronicamente enfermas necessitam compartilhar sentimentos pessoais e serem encorajadas nas suas habilidades em relação aos cuidados dos filhos, reforçando a importância da rede social e do apoio emocional.

Em relação ao quadro de asma infantil, no contato com as mães após 8 meses da finalização do programa, duas mães relataram que seus filhos receberam alta do Ambulatório de Pneumologia (Mãe $1 \mathrm{e}$ Mãe 2) e duas mães relataram que os filhos continuavam em acompanhamento (Mãe 3 e Mãe 4). Cabe aqui apenas observar que as mães em que os filhos haviam recebido alta foram as mesmas mães que não apresentaram níveis de estresse ao final do estudo. Segundo Wrigth e colaboradores (1998), apesar de não haver uma relação causal entre estresse e asma, a complexidade dessa interação indica para a necessidade de novas pesquisas que contemplem aspectos biológicos, psicológicos, socioeconômicos e familiares para se ampliar a compreensão sobre esse tema.

Como estratégia para evitar perdas durante o programa foi oferecida ajuda de custo às participantes em relação ao transporte (pagamento das passagens residência-ambulatório-residência), além de lanche após o término das sessões. Como o programa foi direcionado apenas às mães, as participantes que não tinham com quem deixar seus filhos durante a realização das sessões puderam levar as crianças, ficando essas com um profissional que realizou atividades lúdicas em uma sala ao lado. Acredita-se que tais ações contribuíram para a adesão e desenvolvimento do estudo. Vale ressaltar aqui que as quatro mães possuíam renda familiar entre 1 a 2 salários mínimos e que a situação socioeconômica pode ser tanto um fator dificultador para a adesão em trabalhos de grupo, quanto um fator gerador de estresse (POSSATTI e DIAS, 2002; FERNANDES et al., 2009).

Todas as participantes avaliaram positivamente o programa e relataram que o mesmo contribuiu para diminuição dos seus níveis de estresse. Através de comentários sobre o programa, as mães relataram: Mãe 1 - "Tudo foi perfeito. Pena que acabou. Me ajudou a ficar mais calma e tranquila"., Mãe 2 - "Me ajudou ver a vida diferente, a ver que tudo pode ser diferente. Gostei muito de participar, chegou no momento certo", Mãe 3 - "Eu gostei de tudo, me ensinou muita coisa que eu vou levar para a minha vida toda. Me ajudou a ter controle emocional que eu não conseguia" Mãe 4 - "Achei excelente, mas queria que prorrogasse 0 programa por mais tempo. Gostei dos excelentes conselhos para a melhoria da nossa paciência com os filhos".

Embora o relato de pesquisa tenha possibilitado analisar o programa, não permite generalizações. Limitações metodológicas nesse estudo como a utilização de apenas um instrumento de medida de estresse e a ausência de um grupo controle impossibilitaram uma discussão extensiva e comparação dos resultados, porém, a realização desse estudo e a continuidade do trabalho com essa população devem ser considerados.

\section{CONCLUSÃO}

Nossos dados demonstraram que a participação das mães no programa pode influenciar positivamente nos níveis de estresse das participantes. Após a realização desse estudo, o programa foi reavaliado e estendido a outras mães do ambulatório de Pneumologia e acredita-se que sua continuidade será um grande desafio que trará benefícios tanto para saúde das mães, quanto para a saúde das crianças. Vale destacar que esse foi um primeiro estudo que apresentou um programa com mães de crianças com asma e seus resultados preliminares reforçam a necessidade de novos estudos sobre o tema e a importância da implementação de programas de intervenção para controle e redução de estresse que incluam mães e cuidadores.

\section{FINANCIAMENTO}

CAPES - Coordenação de Aperfeiçoamento de Pessoal de Nível Superior. 


\section{REFERENCIAS}

1. BELLIN MH, KUB J, FRICK KD, et al. Stress and quality of life in caregivers of inner-city minority children with poorly controlled asthma. Journal of Pediatric Health Care, 2013; 27(2): 123-34.

2. CAIRO S, SANT'ANNA CC. Estresse em mães e cuidadoras de crianças e adolescentes com asma: um estudo sobre a frequência do estresse e fatores estressores. Caderno Saúde Coletiva, 2014; 22(4): 393-400.

3. CASTRO EK, PICCININI CA. Implicações da doença orgânica crônica na infância para as relações familiares: algumas questões teóricas. Psicologia Reflexão e Crítica, 2002; 15(3): 625-35.

4. CELANO MP, HOLSEY CN, KOBRYNSKI LJ. Home-based family intervention for low-income children with asthma: A randomized controlled pilot study. Journal of Family Psychology, 2012; 26(2): 171-78.

5. CROWE TK, VANLEIT B, BERGHMANS KK. Mothers' perceptions of child care assistance: The impact of a child's disability. American Journal of Occupational Therapy, 2000; 54(1): 52-58.

6. DSBP - DIRETRIZES DA SOCIEDADE BRASILEIRA DE PNEUMOLOGIA E TISIOLOGIA PARA O MANEJO DA ASMA - 2012. Jornal Brasileiro de Pneumologia, 2012; 38 (Supl.1): S1-S46.

7. FERNANDES LFB, LUIZ AMAG, MIYAZAKI MCOS, et al. Efeitos de um programa de orientação em grupo para cuidadores de crianças com transtornos psiquiátricos. Estudos de Psicologia (Campinas), 2009; 26(2), 147-158.

8. GLOBAL ASTHMA REPORT 2014. Auckland, New Zealand, Global Asthma Network, 2014. Disponível em: http://www.globalasthmareport.org/ resources/Global_Asthma_Report_2014.pdf

9. HENDRICKS-FERGUSON VL, PRADHAN K, SHIH CS, et al. Pilot Evaluation of a Palliative and Endof-Life Communication Intervention for Parents of Children With a Brain Tumor. Journal of Pediatric Oncology Nursing, 2017; 34(3), $203-213$.

10. KAUGARS AS, KLINNERT MD, BENDER BG. Family influences on Pediatric Asthma. Journal Pediatric Psychology, 2004; 29(7): 475-91.

11. KUSCHNIR FC, GURGEL RQ, SOLÉ D, et al. ERICA: prevalência de asma em adolescentes brasileiros. Revista Saúde Pública, 2016; 50(Supl.1): 13s.

12. LIPP MEN. (organizadora). Mecanismos Neuropsicofisiológicos do Stress: teoria e aplicações clínicas. 3ª edição. São Paulo: Casa do Psicólogo, 2003; p. 17 e 107.

13. LIPP, MEN. Manual do Inventário de Sintomas de Stress para adultos de Lipp (ISSL) 3a. ed. São Paulo: Casa do Psicólogo, 2005.

14. MAHMOUD S, ELAZIZ NAA. Effect of psycho-educational training program for parent's having child with leukemia on their experience and psychological wellbeing. Journal of Education and Practice, 2015; 6(12): 13-29.

15. MATSUKURA TS, MARTURANO EM, OISHI J, et al. Estresse e suporte social em mães de crianças com necessidades especiais. Revista Brasileira Educação Especial, 2007; 13(3): 415-28.

16. MINOR HG, CARLSON LE, MACKENZIE MJ, et al. Evaluation of a mindfulness-based stress reduction (MBSR) program for caregivers of children with chronic conditions. Social Work in Health Care, 2006; 43(1): 91-109.

17. PARENTE AAAI, FARIAS LS, MARCH MFBP, et al. Uso de espaçadores em crianças com asma: emprego da técnica inalatória. Revista Eletrônica Acervo Saúde, 2012; 4(2), 260-268.

18. PELLETIER L, GODIN G, LEPAGE L, et al. Social support received by mothers of chronically ill children. Child: Care, Helth and Development, 1994; 20: 115-31.

19. POSSATTI IC, DIAS MR. Multiplicidade de Papéis da Mulher e seus Efeitos para o Bem-Estar Psicológico. Psicologia: Reflexão e Crítica, 2002; 5(2): 293-301.

20. SALOMÃO JÚNIOR JB, MIYAZAKI MCOS, CORDEIRO JA, et al. Asma, competência social e transtornos comportamentais em crianças e adolescentes. Estudos Psicologia (Campinas), 2008; 25(2):185-92.

21. SABMANN H, HAIR M, DANNE T, et al. Reducing stress and supporting positive relations in families of young children with type 1 diabetes: A randomized controlled study for evaluating the effects of the DELFIN parenting program. BMC Pediatrics, $2012 ; 12: 152$.

22. SILVER EJ, STEIN REK., DADDS MR. Moderating effects of family structure on the relationship between physical and mental health in urban children with chronic illness. Journal of Pediatric Psychology, 1996; 21(1), 43-56.

23. TANGANELLI MS (2003). O treino de controle de stress aplicado a mulheres. In: Lipp MEN (org.). Mecanismos Neuropsicofisiológicos do Stress: teoria e aplicação clínica. 3a ed., 2003, p. 205. São Paulo: Casa do Psicólogo.

24. TSIOULI E, PAVLOPOULOS V, ALEXOPOULOS EC, et al. Short-term impact of a stress management and health promotion program on perceived stress, parental stress, health locus of control, and cortisol levels in parents of children and adolescents with diabetes type 1: A pilot randomized controlled trial. Explore, 2014; 10(2): 88-98.

25. WARNER CM, LUDWIG K, SWEENEY C, et al. Treating persistent distress and anxiety in parents of children with cancer: An initial feasibility trial. Journal of Pediatric Oncology Nursing, 2011; 28(4): 224-30.

26. WRIGHT RJ, RODRIGUEZ M, COHEN S. Review of psychosocial stress and asthma: an integrated biopsychosocial approach. Thorax, 1998; 53:1066-1074.

27. WRIGHT RJ, COHEN S, CAREY V, et al. Parental Stress as a Predictor of Wheezing in Infancy - A Prospective Birth-Cohort Study. American Journal of Respiratory and Critical Care Medicine, 2002; 165: 358-365.

28. YEH HY, MA WF, HUANG JL, et al. Evaluating the effectiveness of a family empowerment program on family function of children with asthma: A randomized control trial. International Journal of Nursing Studies, 2016; 60: 133-144. 